\title{
Discussion of Normal University Students' SMK and the Development of MPCK
}

\author{
Mengmin Guo, a and Sanping $\mathrm{Li}^{1, \mathrm{~b}}$ \\ ${ }^{1}$ School of Mathematics and Information Science, Shaanxi Normal University, Xi'an, China \\ agmm24@126.com, blisanping@snnu.edu.cn
}

Keywords: MPCK; Normal students of mathematics major; MK; SMK

\begin{abstract}
The transformation from mathematics knowledge (MK) to mathematics pedagogical content knowledge (MPCK) is an important channel for mathematics teachers to develop their MPCK, and in order to reach the target, they must firstly have a good grasp of mathematics knowledge. While in the survey about mathematical normal students' level of subject matter knowledge (SMK) in the discipline of simple logic, we could see that normal university students have a low level of it, which indirectly shows that they are quite weak in their MK. Thus, we should not only realize the magnitude of general pedagogical knowledge and content knowledge, but also attach great importance to MK in the development of normal students' MPCK.
\end{abstract}

\section{浅谈师范生 SMK 与 MPCK 的发展}

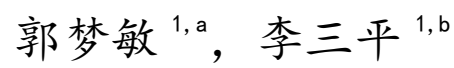 \\ 1. 陕西师范大学 数学与信息科学学院, 中国陕西西安, 710119 \\ gmm24@126.com, ${ }^{\mathrm{a}}$ lisanping@snnu.edu.cn
}

摘要: 数学教师的数学教学内容知识 (Mathematical Pedagogical Content Knowledge, 简称 MPCK) 发展的重要途径是实现由数学学科知识 (Mathematical Knowledge, 简称 MK) 向 MPCK 的转化, 数学教师要实现这个转化, 必须首先具备扎实深厚的 MK. 而对数学专业师 范生在中学简易逻辑方面学科内容知识(Subject Matter Knowledge, 简称 SMK) 的掌握情况的 调查分析看到, 师范生的 SMK 水平不高, 间接反映了他们的 MK 较为薄弱. 因此, 在师范 生 MPCK 的发展中, 不仅要强调一般教学法 (Pedagogical Knowledge, 简称 PK) 与有关数学 学习知识 (Content Knowledge, 简称 CK) 的重要作用, 更要回归基础, 重视 MK 的基础性 与重要性.

关键词: MPCK; 数学师范生; MK; SMK

\section{1. 引言}

近年来, 随着对数学教师专业知识的深入研究, 一种融合了数学学科知识 (Mathematical Knowledge, 简称 MK)、一般教学法知识 (Pedagogical Knowledge, 简称 PK) 与有关数学学习 的知识 (Content Knowledge, 简称 CK) 的数学教师专业知识类型——数学教学内容知识 (Mathematical Pedagogical Content Knowledge，简称 MPCK）愈发受到重视。MPCK 作为数 学教师从事数学教学所应具备的核心知识, 它不仅是数学教师专业性的重要体现, 也是数学 教师专业发展的新的生长点 [1], 故 MPCK 成为了师范教育的一个重要的风向标。

在教学中, 教师往往需要综合运用 $\mathrm{MK} 、 \mathrm{PK}$ 与 $\mathrm{CK}$ 这三类知识, 才可能把科学形态的数学内 容有效地转化为教育形态的数学知识, 才可能达到帮助学生理解数学的目的 [2]。掌握科学形 式的数学知识是有效转化的前提, 这需要教师具有深厚扎实的 $\mathrm{MK}$; 有效转化是达到教育形 态的数学知识的重要途径, 这需要教师有娴熟扎实的 $\mathrm{PK}$ 与 $\mathrm{CK}$ 。

在我国目前的高等师范教育中, 一些院校在完成数学专业知识传授的同时, 更偏重于教授 “如 
何有效实现知识形态转化” 的 PK 与 CK; 而且, 部分数学师范生由于从高等数学专业课学习 中难以找到与中学数学相对接的学科知识, 以及自身相关教育、教学知识的欠缺, 他们更倾 向于 PK 与 $\mathrm{CK}$ 的学习。高中数学教师的金字塔知识结构图 [3] 突出了 $\mathrm{MK}$ 对于发展 PK 与 $\mathrm{CK}$ 的基础地位, 而目前数学师范生 $\mathrm{MK}$ 的实然水平究竟怎样呢? 是否具备了促进 PK 与 $\mathrm{CK}$ 发 展的坚实基础呢?

由于学科内容知识(Subject Matter Knowledge, 简称 SMK) 是 MK 较为基础的部分, 本文针对 数学专业师范生 MK 维度之一的 SMK 展开了一项调查, 我们选取了简易逻辑中易使人困惑 的 “命题的否定” 与 “逆否命题” 这两个知识点, 以探究数学师范生 SMK 的实然水平, 间 接了解他们的 MK 情况, 希望为更好地促进数学专业师范生 MPCK 发展提供一些建议。

\section{2. 调查的实施}

\section{1. 调查的对象}

以陕西省某师范大学 2013 级数学专业在校大三师范生为调查对象: 他们已完成大学三年的数 学专业课学习, 有一定的数学专业知识功底。

2.2. 调查的方法与过程

为了深入了解师范生简易逻辑的 SMK, 我们设置了三个相关测试题. 测试题 1 主要考查师范 生对含有全称量词的 “若 $P$ 则 $Q$ ” 命题的否定（或非命题）的掌握情况; 测试题 2 和测试题 3 主要考查师范生对命题的逆否命题相关知识的掌握情况. 我们分别在各班专业选修课授课 期间发放测试题，并于课后回收，回收率 100\%。

\section{3. 调查结果及其分析}

3. 1 . 师范生对命题的否定掌握情况

为了解数学专业师范生对 “若 $P$ 则 $Q$ ” 的否定命题的掌握情况, 我们设置了测试题 1: 判断命题 “若 $x^{2}=1$, 则 $x=-1$ ” 的真假, 并写出该命题的否定命题。

该命题是假命题, 且该命题的否定是 “存在 $x$, 使得 $x^{2}=1$ 但 $x \neq-1$ ”。 在调查的 180 名师范生中, 九成以上的均能对命题的真假正确判断, 但在对原命题做出假命 题判断的同学中, 有 26 名对象也对该命题的否定做出了真假判断, 其中 11 名同学认为该命 题的否定仍是假命题。这间接说明了有些师范生对命题的否定的理解还不到位。

从表 1 可以看到, 近三成调查对象正确回答了测试题 1 的否定命题形式, 并且仅有 15\%的同 学给出了完全正确的答案, $14.44 \%$ 的同学尽管在该命题的否定中对隐含的全称量词做出了否 定, 但是却对 “ $\neg(P \rightarrow Q) \Leftrightarrow \neg(\neg P \vee Q) \Leftrightarrow P \wedge \neg Q$ ” 这一逻辑等价关系不清楚, 以至于给出 了形如 “存在 $x^{2}=1$, 则 $x \neq-1$ ”、“存在 $x^{2}=1$, 有 $x \neq-1$ ” 等类似答案。近七成同学的答案 是错误的, 并且 $57.78 \%$ 的同学认为该命题的否定是 “若 $x^{2}=1$, 则 $x \neq-1$ ”, 简单地将 “若 $P$ 则 $Q$ ” 型命题的否定理解为保留条件否定结论的 “若 $P$ 则非 $Q$ ” 形式, 这在一定程度上说明 尽管大部分数学师范生能够区分命题的否定与否命题这两个概念, 但对这两个概念的辨别停 留在是否否定条件的较浅层次，并未深入理解与掌握简易逻辑的 SMK。 
表 1 测试题 1 命题的否定的作答形式统计表

\begin{tabular}{lcc}
\hline 逆否命题的形式 & $\begin{array}{c}\text { 人 } \\
\text { (人) }\end{array}$ & $\begin{array}{c}\text { 数 } \\
\text { (所 占 百 分 比 } \\
(\%)\end{array}$ \\
\hline 存在 $x$, 使得 $x^{2}=1$ 但 $x \neq-1$ & 27 & $15.00 \%$ \\
形如 “存在 $x^{2}=1$, 则 $x \neq-1$ ”、“存在 $x^{2}=1$, 有 $x \neq-1$ ” 26 & $14.44 \%$ \\
等 & 104 & $57.78 \%$ \\
若 $x^{2}=1$, 则 $x \neq-1$ & 23 & $12.78 \%$ \\
其他 & & \\
\hline
\end{tabular}

\section{2. 数学专业师范生对命题的逆否命题掌握情况}

在了解数学专业师范生对命题的逆否命题的掌握情况方面, 我们设置了两道测试题:

测试题 2: 判断命题 “若 $a<b$, 则 $a^{2}<b^{2}$ ” 的真假, 并写出其逆否命题;

测试题 3: 判断命题 “若 $x>-1$, 则 $x^{2} \geq 0$ ” 的真假, 并写出其逆否命题。

在回答测试题 2 的 56 名同学中，全部均给出了该命题是假命题的正确判断，并且 96.43\%的 同学正确地给出了该命题的逆否命题 “若 $a^{2} \geq b^{2}$, 则 $a \geq b$ ”, 这在一定程度上说明了绝大部 分的同学掌握了逆否命题的相关知识. 然而, 对回答测试题 3 的 124 名同学分析发现, 情况 却不容乐观。测试题 3 中的原命题显然是真命题, 但仍有 $8.87 \%$ 的同学不能做出正确判断。 对作出错误判断的部分答卷分析可见, 他们习惯于从判断逆否命题的真假去推断原命题的真 假。这反映了小部分师范生解题思维较为僵化，不能灵活变通。

表 2 测试题 3 逆否命题作答形式统计表

\begin{tabular}{llc}
\hline 逆否命题的形式 & 人数（人） & $\begin{array}{c}\text { 所 占 百 分 比 } \\
(\%)\end{array}$ \\
\hline 若 $x^{2}<0$, 则 $x \leq-1$ & 105 & $84.68 \%$ \\
若 $x^{2}$ 不大于等于 0 , 则 $x$ 不大于等于 -1 & 1 & $0.81 \%$ \\
含存在量词的形式 & 7 & $5.64 \%$ \\
其他 & 11 & $8.87 \%$ \\
\hline
\end{tabular}

从表 2 可见, $84.68 \%$ 的同学认为逆否命题是 “若 $x^{2}<0$, 则 $x \leq-1$ ”, 按照 “若 $P$ 则 $Q$ ” 的逆 否命题为 “若 $\neg Q$ 则 $\neg P$ ” 的角度, 答案确是如此, 但具体到这道题目, 当 $x^{2}<0$ 时, $x$ 连实 数都不是 (即不等式 $x^{2}<0$ 无实数解), 所以, 在实数范围内只能肯定它不是 “大于 -1 ” 的 实数 [4]。故该形式尽管符合逆否命题的形式, 但其逆否逆否命题却应表述为 “若 $x^{2}$ 不大于 等于 0 , 则 $x$ 不大于等于 -1 ” [4], 在 124 名同学中仅有 1 人考虑到上述情况并正确作答。这 在一定程度上反映出大部分数学专业师范生仅满足于形式上的正确, 却忽略了对逆否命题内 容可行性的深层次分析。在对答卷统计分析中，我们还发现，5.64\%的调查对象在该命题的逆 否命题中使用了存在量词, 一方面说明了他们意识到原命题含有全称量词, 另一方面却反映 了他们对 “全称命题的逆命题、逆否命题等四种形式都是全称命题” 认识的盲区, 并混淆 “若 $P$ 则 $Q ”$ 的非命题与逆否命题中的量词关系。

\section{4. 结论与建议}

从上述对数学专业师范生在简易逻辑 SMK 掌握情况的分析可见, 尽管大部分师范生掌握了基 本的命题逻辑与谓词逻辑的相关知识, 但学科知识方面却不够深入和系统, 对关键内容知识 的理解较为肤浅、对易混淆知识点也存在含混现象。这在一定程度上说明了数学专业师范生 
SMK 的水平属于较低层次，也反映了他们 MPCK 的基础部分一一 MK 是较为薄弱的。

MPCK 不仅体现了数学教师专业的独特性, 也是数学教师专业发展的核心 [5], 数学教师 MPCK 发展的重要途径是实现由 MK 向 MPCK 的转化 [6]。数学教师要实现这个转化, PK 与 $\mathrm{CK}$ 的催化固不可少, 但必须具备扎实深厚的 $\mathrm{MK}$ 。有效数学教学的核心要素是教师知识 [7], 而只有精通数学学科知识, 才能在数学教学中高屋建瓴、举一反三 $[8]$ 。为此, 一方面, 高师 院校在数学专业课设置与课程结构应加强与中学数学的联系, 培养数学专业师范生运用高观 点分析解释中学数学的意识, 给他们提供一个强调 $\mathrm{MK}$ 基础性与重要性的学习环境。相关研 究表明 [9], 数学专业师范生学科知识欠佳的原因并非所学的数学专业课不够系统深入, 主要 症结是这些课程的内容与中学数学之间缺乏关联。而在相关课程设置中强化高等数学与初等 数学之间的联系，能在一定程度上改善师范生学科知识欠佳的状况。

另一方面, 数学专业师范生应树立正确的学科内容知识观念, 通过专业学习、课后探索以及 教育实践, 积极主动地建立起专业课知识与中学数学学科知识间的关系, 不断提升自身的学 科内容知识, 夯实 $\mathrm{MK}$ 的基础成分。当然, $\mathrm{MK}$ 的其他三个维度一一数学观念、数学思想方 法与数学史知识也是师范生需要着力学习的。除此之外, 数学专业师范生应建立起 MK、PK 与 $\mathrm{CK}$ 三者相互促进、共同进步的意识，在深厚扎实的 $\mathrm{MK}$ 的基础上不断丰富与完善 $\mathrm{PK}$ 与 $\mathrm{CK}$, 在夯实 $\mathrm{PK}$ 与 $\mathrm{CK}$ 的氛围中持续深化学习 $\mathrm{MK}$ 。 MPCK 并非仅是 $\mathrm{MK} 、 \mathrm{PK}$ 与 $\mathrm{CK}$ 的简单 叠加和随机组合, 而是不同知识要素交汇融合的产物, 并在融合过程中改变各要素的形态与 彼此间的关系 $[10]$ 。通过这三者在相互促进中彼此融合, 在彼此融合中相互促进, 能在一定 程度上加速数学专业师范生 MPCK 的生成。

\section{5. 结语}

数学专业师范生 MPCK 的发展过程中, 不仅需要认识到 PK 与 $\mathrm{CK}$ 对科学形式的数学向教育 形态的数学知识有效转化的重要作用, 同时也应回归基础, 注重学科内容知识的地位, 强化 $\mathrm{MK}$ 的深入系统学习, 并通过这三者的相互促进达到彼此间的有机融合, 进而加速数学专业 师范生 MPCK 的发展进程。

\section{6. 致谢}

特别感谢陕西师范大学研究生院项目（No. GERP-15-40）对本研究提供的帮助。

\section{参考文献}

[1] 童莉. 数学教师专业发展的新视角--数学教学内容知识(MPCK)[J]. 数学教育学报, 2010(2): 23-27.

[2] 黄毅英, 许世红. 数学教学内容知识--结构特征与研发举例 [J]. 数学教育学报, 2009(1): 5-9.

[3] 刘俊华, 胡典顺, 纪静萍, 等. 高中数学教师 MPCK 发展的调查研究[J]. 数学教育学报, 2015(1): 45-50.

[4] 罗增儒. 关于“取值范围”讨论之我见（再续） [J]. 中学数学教学参考, 2014(7): 59-63.

[5] 李渺, 宁连华. 数学教学内容知识(MPCK)的构成成分表现形式及其意义[J]. 数学教育学 报, 2011(2): 10-14.

[6] 胡典顺. 教师 MPCK 发展的实证研究[M]. 北京：科学出版社, 2015, 152-157.

[7] M. Walshaw. 2012. "Teacher knowledge as fundamental to effective teaching practice," Journal of Mathematics Teacher Education, 15(3): 181-185.

[8] 王子兴. 论数学教师专业化的内涵[J]. 数学教育学报, 2002(4): 63-67. 
[9] 赵冬臣，马云鹏. 从教师专业知识状况看教师教育课程改革[J]. 课程·教材·教法, 2011(10): $18-23$.

[10]王宏, 史宁中. 基于教师专业发展视角的数学教学内容知识研究[J]. 东北师大学报（哲学 社会科学版)，2015(6): 244-248.

\section{Acknowledgement}

The authors are grateful to the support of the foundation of graduate school of Shaanxi Normal University (No.GERP-15-40).

\section{References}

[1] Tong Li. 2010. "Mathematical Pedagogical Content Knowledge: the New Perspective of Mathematics Teacher Professional Development," Journal of Mathematics Education, 19(2): 23-27.

[2] Wong Ngaiying \& $\mathrm{Xu}$ Shihong. 2009. "Mathematical Pedagogical Content Knowledge--Structure and Research Case,” Journal of Mathematics Education, 18(1): 5-9.

[3] Liu Junhua, HU Dian-shun, Ji Jing-ping, et al. 2015. "Study on MPCK Development of Senior Mathematics Teacher," Journal of Mathematics Education, 24(1): 45-50.

[4] Luo Zengru. 2014. "My Opinions on the Discussion about Value Range," Teaching Reference of Middle School Mathematics, 7: 59-63.

[5] Li Miao \& Ning Lianhua. 2011. "Study about the Component and Form of Mathematics Pedagogical Content Knowledge and Its Meaning," Journal of Mathematics Education, 20(2): $10-14$.

[6] Hu Dianshun. 2015. “The Empirical Study on the Development of Teachers' MPCK," Beijing: Science Press, 152-157.

[7] M. Walshaw. 2012. "Teacher knowledge as fundamental to effective teaching practice," Journal of Mathematics Teacher Education, 15(3): 181-185.

[8] Wang Zixing. 2002. "On the Connotation and Sense of Mathematics Teaching as a Profession," Journal of Mathematics Education, 11(4): 63-67.

[9] Zhao Dongchen \& Ma Yunpeng. 2011. "Examine the Curriculum Reform of Teacher Education from the Perspective of Teachers' Professional Knowledge," Curriculum, Teaching Material and Method, 31(10): 18-23.

[10]Wang Hong \& Shi Ningzhong. 2015. "On the Pedagogical Content Knowledge of Mathematics from the Perspective of Teacher Professional Development," Journal of Northeast Normal University (Philosophy and Social Sciences), 6: 244-248. 